\title{
The effects of low-repetition and light-load power training on bone mineral density in postmenopausal women with sarcopenia: a pilot study
}

Kanako Hamaguchi', Toshiyuki Kurihara', Masahiro Fujimoto', Motoyuki lemitsu', Koji Sato², Takafumi Hamaoka ${ }^{3}$ and Kiyoshi Sanada ${ }^{1 *}$ (D)

\begin{abstract}
Background: Age-related reduction in bone mineral density (BMD) is generally accelerated in women after menopause, and could be even more pronounced in individuals with sarcopenia. Light-load power training with a low number of repetitions would increase BMD, significantly reducing bone loss in individuals at risk of osteoporosis. This study investigated the effects of low-repetition, light-load power training on BMD in Japanese postmenopausal women with sarcopenia.
\end{abstract}

Methods: The training group $(n=7)$ followed a progressive power training protocol that increased the load with a weighted vest, for two sessions per week, over the course of 6 weeks. The training exercise comprised five kinds of exercises (squats, front lunges, side lunges, calf raises, and toe raises), and each exercise contained eight sets of three repetitions with a 15-s rest between each set. The control group $(n=8)$ did not undergo any training intervention. We measured BMD, muscle strength, and anthropometric data.

Results: Within-group changes in pelvis BMD and knee extensor strength were significantly greater in the training group than the control group ( $p=0.029$ and 0.030 for pelvis BMD and knee extensor strength, respectively). After low-repetition, light-load power training, we noted improvements in pelvis BMD (1.6\%) and knee extensor strength (15.5\%). No significant within- or between-group differences were observed for anthropometric data or forearm BMD.

Conclusions: Six weeks of low-repetition, light-load power training improved pelvis BMD and knee extensor strength in postmenopausal women with sarcopenia. Since this training program does not require high-load exercise and is therefore easily implementable as daily exercise, it could be an effective form of exercise for sedentary adults at risk for osteoporosis who are fearful of heavy loads and/or training that could cause fatigue.

Trial Registration: This trial was registered with the University Hospital Medical Information Network on 31 October 2016 (UMIN000024651).

Keywords: Power training, Bone mineral density, Sarcopenia, Postmenopausal women

\footnotetext{
* Correspondence: ksanada@fc.ritsumei.ac.jp

${ }^{1}$ College of Sport and Health Science, Ritsumeikan University, Shiga, Japan

Full list of author information is available at the end of the article
} 


\section{Background}

Osteoporosis, a chronic disease involving reduced bone mineral density (BMD) [1], is one of the most prevalent factors contributing to fractures among elderly individuals $[1,2]$. Age-related reduction in BMD is generally accelerated in women after menopause [3]. In addition, sarcopenia, characterized by age-related muscle atrophy [4] and weakness [5], is a primary factor influencing muscle performance [6] and is associated with an increased risk of osteoporosis [7]. An effective exercise training program to preserve BMD is needed for postmenopausal women with sarcopenia.

High-velocity resistance training, also referred to as power training, is reportedly more effective than conventional strength training for preventing osteoporosis $[8,9]$. Stengel et al. [8] found that 1 year of periodised power training, designed such that 12 weeks of high-load training (70-90\% $1 \mathrm{RM}$ ) was interleaved by $4-5$ weeks of low-load training (50\% $1 \mathrm{RM}$ ), was more effective in reducing bone loss in postmenopausal women than strength training [8]. Another study found that high-load and lowrepetition power training (i.e., four sets of 3-5 repetitions with fast lifts at $85-90 \%$ of $1 \mathrm{RM}$ ) was effective in preserving BMD in postmenopausal women with osteoporosis or osteopenia [9]. Thus, power training appears to be more effective than strength training in reducing bone loss in individuals at risk for osteoporosis.

Although these previous studies employed high-load power training, some studies have found that maximal power output is achieved at $30-70 \%$ of 1 RM [10-12], suggesting that similar power output can be generated with a lighter load. A previous study found that peak power during light-load (35\% of $1 \mathrm{RM}$ ) power training was comparable to or even larger than that of high-load (70\% of $1 \mathrm{RM}$ ) power training [11]. It seems that even though the generated force output was smaller, the lighter load allowed subjects to move more quickly, resulting in greater power output. Light-load power training with a weighted vest in older postmenopausal women has been shown to increase hip BMD, demonstrating its effectiveness in preventing significant bone loss [13]. Therefore, with regard to preventing bone loss, light-load power training would likely serve as a better form of exercise training than high-load power training.

In addition to load conditions, the number of repetitions also affects muscle power output. Maximizing muscle power output throughout the training session is critical for bone adaptation, for which sufficient rest intervals between sets are required. When the total volume of exercise and rest interval between sets is equal, a lowrepetition protocol provides more rest intervals than a high-repetition protocol, thereby restoring one's ability to generate muscle power [14]. Taken together, lowrepetition and light-load power training would increase
$\mathrm{BMD}$, reducing significant bone loss in postmenopausal women with sarcopenia who are at risk for osteoporosis. However, to our knowledge, no study has investigated the effects of low-repetition, light-load power training on BMD in these women.

Against this backdrop, the objective of this study was to investigate the effects of low-repetition, light-load power training on BMD in Japanese postmenopausal women with sarcopenia. Subjects were allocated to either the 6-week training group or control group. We hypothesized that the training group would exhibit greater increases in BMD than the control group.

\section{Methods}

\section{Subjects}

This study recruited middle-aged postmenopausal women with sarcopenia from local communities to perform low-repetition, light-load power training. Women who were younger than 65 years of age and at least 2 years past menopause were included. None of the subjects had cardiovascular, metabolic, or orthopaedic disease, on-going medication, unstable medical conditions, or had experienced a fracture in the past 6 months. Those who were engaged in regular exercise sessions at the time of the study were also excluded. Skeletal muscle mass index (SMI) was determined by DXA scan, and individuals with SMI $<6.12 \mathrm{~kg} / \mathrm{m}^{-2}$ (the reference value for classifying sarcopenia class 1 for Japanese women based on EWGSOP criteria $[5,15])$, were diagnosed with sarcopenia and included in the study. In total, 19 postmenopausal women with sarcopenia were included. All subjects were informed of the benefits and risks of the investigation prior to providing written informed consent. This study was approved by the Ethics Committee of Ritsumeikan University (Approval number: BKC-IRB-2012-032). All the participants reviewed and signed an informed consent form, in accordance with the Declaration of Helsinki.

\section{Study design and intervention}

Subjects were initially allocated to either the training group or control group following a 1:1 ratio. Two subjects allocated to the training group declined to participate in exercise sessions and therefore were treated as dropouts, which resulted in eight and nine subjects in the training and control groups, respectively. The training program comprised five exercises (squats, front lunges, side lunges, calf raises, and toe raises). To accentuate power production, the concentric phase was performed as fast as possible while still maintaining the proper posture (Fig. 1). For each exercise, subjects were asked to perform eight sets of three repetitions with a 15 -s rest between each set to restore mechanosensitivity of bone cells and enhance the osteogenic effect of exercise [16]. Each exercise session 


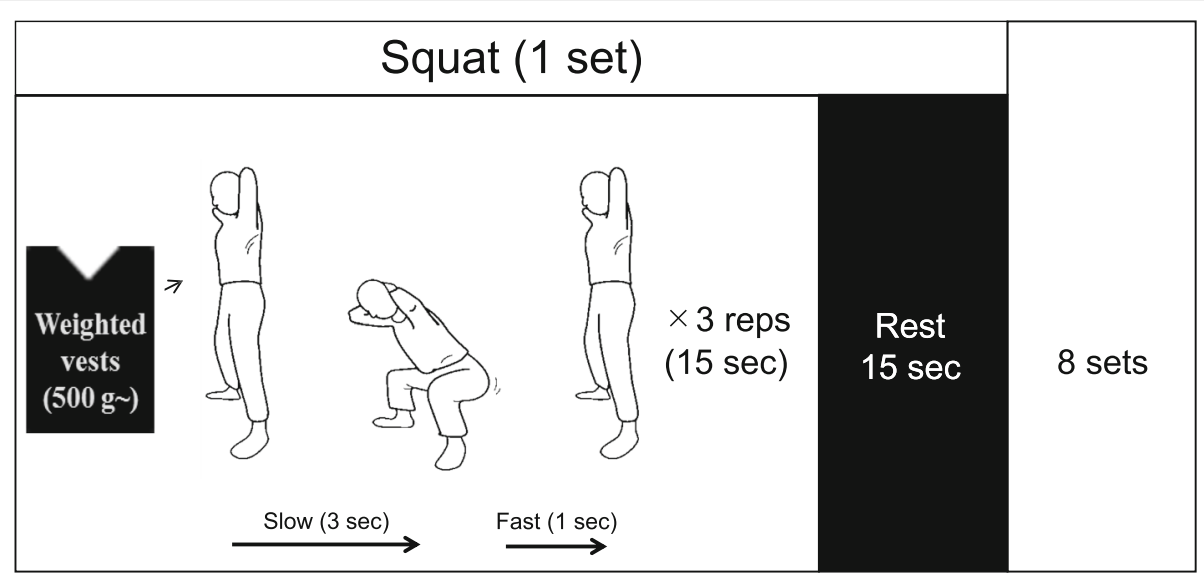

Fig. 1 Illustration of the low-repetition and light-load power training in this study. Similar sequences were performed with four other exercises (front lunge, side lunge, calf raise, toe raise). The training group followed a progressive power training protocol that increased intensity with a weighted vest over 6 weeks, comprising 2 sessions each week

lasted approximately $60 \mathrm{~min}$, including the warm-up and cool-down portions, which consisted of stretching and cycling exercises. The training group followed a progressive power training protocol with a weighted vest that increased in intensity over 6 weeks, comprising two sessions each week. During the first session of the power training program, subjects wore the vest without any weight in order to familiarize themselves with the training procedures. Perceived exertion was measured by the Borg Scale after each exercise to determine whether weight could be added or not; if the subject was capable of completing all eight sets, the weight was increased by $380-760 \mathrm{~g}$ in the next session. The weight was reduced or kept the same in the subsequent session if the subject's perceived exertion exceeded 13 on the Borg scale ("somewhat hard" level) or if the subject complained of any muscle pain. All training sessions were conducted at the laboratory under direct supervision. Control subjects were instructed to maintain their daily physical activity level.

\section{Measurements}

BMD (arm, spine, pelvis, leg, and total body), SMI, and muscle strength were measured at baseline and after the 6-week intervention (hereafter, Pre and Post, respectively). Subjects were given 3-7 days of rest before the post-training assessment. Dietary intake was also measured using the brief-type self-report diet history questionnaire (BDHQ).

\section{DXA scan}

BMD, SMI, and body composition were assessed by DXA with EnCore software (Lunar Prodigy, GE Healthcare, UK). Subjects fasted overnight and did not perform any exercise in the morning before measurements. Total body and regional BMD, bone mineral content (BMC), fat mass, and fat-free mass (FFM) were analysed. All scans were performed and analysed by a single trained and licensed technician who was blinded to the group allocation. SMI was calculated as follows:

$$
\begin{aligned}
\operatorname{SMI}\left(\mathrm{kg} / \mathrm{m}^{2}\right)= & \text { appendicular skeletal muscle mass }(\text { FFM-BMC }) \\
& /(\text { body height })^{2}
\end{aligned}
$$

\section{Muscle strength}

Maximum isometric strength of the knee extensor (KE strength) on the right side was measured using a dynamometer (Biodex System 4, Sakai Med, Japan). Subjects were seated with knees flexed at $90^{\circ}$ and hips flexed at approximately $80^{\circ}$ [17]. Maximum voluntary contraction was performed twice, for $5 \mathrm{~s}$ each, with $1 \mathrm{~min}$ of rest between them. The maximum value was regarded as isometric muscle strength. Strength was recorded in Newton • meters $(\mathrm{Nm})$ and normalized by body mass $(\mathrm{Nm} / \mathrm{kg})$. Hand grip strength (grip strength) was also assessed for each hand by a hand grip dynamometer.

\section{Statistical analysis}

Outcome measures were BMD (arm, spine, pelvis, leg, and total body), SMI, and muscle strength. Potential differences between the control and training groups at baseline were assessed by independent $t$-tests. A two-way, mixed-design ANOVA, with group (control and training) and time (pre and post) as between- and within-subject factors, was performed to determine main and interaction effects on outcome measures. When significant interactions were identified, the Bonferroni post-hoc test was performed to detect the sources of significant differences. Within-group changes (\% change) between baseline (Pre) and follow-up (Post) in BMD and muscle strength were calculated and an independent $t$-test was performed to examine if relative changes from baseline to follow-up 
were significantly different between the control and training groups. Within-group change was calculated as follows:

$\%$ change $=($ Post-value-Pre-value $) /$ Pre-value $\times 100$

All analyses were performed using IBM SPSS (version 19). Statistical significance was set at $\alpha<0.05$.

\section{Results}

\section{Subject characteristics, attendance, and training}

Of the initial 17 subjects, 15 completed all baseline and follow-up testing; one subject each from the training and control groups dropped out before follow-up testing for personal reasons unrelated to the study. Baseline characteristics of subjects in the training $(n=7$, mean age $=60.4 \pm 2.7$ years $)$ and control $(n=8$, mean age $=60.6 \pm 2.4$ years) groups are presented in Table 1 . There were no significant differences between groups in age, postmenopausal period, BMI, or nutritional intake $(p>0.05)$. Subjects in the training group demonstrated a high level of adherence to the exercise intervention with a participation rate above 92\%, with the exception of the one subject who dropped out during the intervention. No adverse events, such as falls, fractures, or bodily pain, were reported during the 6-week intervention. The final session of power training was conducted in a manner such that perceived exertion scores were between 10 and 13 on the Borg Scale (RPE: $11.4 \pm 0.8$, added weights: $3.5 \pm 0.8 \mathrm{~kg}$ ).

\section{Bone mineral density (BMD)}

Table 2 shows baseline (Pre) and follow-up (Post) values for BMD. Corresponding $\mathrm{F}$ and $P$ values of the time $\times$

Table 1 Subject characteristics

\begin{tabular}{llll}
\hline & Training $(n=7)$ & Control $(n=8)$ & $P$ \\
\hline Age $(\mathrm{yrs})$ & $60.4 \pm 2.7$ & $60.6 \pm 2.3$ & .86 \\
Postmenopausal period $(\mathrm{yrs})$ & $11.1 \pm 6.3$ & $8.9 \pm 4.1$ & .43 \\
Height $(\mathrm{cm})$ & $157.0 \pm 4.3$ & $157.6 \pm 5.0$ & .82 \\
Weight $(\mathrm{kg})$ & $47.4 \pm 5.0$ & $48.8 \pm 4.4$ & .60 \\
BMI $\left(\mathrm{kg} / \mathrm{m}^{2}\right)$ & $19.2 \pm 1.2$ & $19.7 \pm 1.8$ & .58 \\
SMI $\left(\mathrm{kg} / \mathrm{m}^{2}\right)$ & $5.4 \pm 0.3$ & $5.6 \pm 0.5$ & .52 \\
SBP $(\mathrm{mmHg})$ & $129 \pm 26$ & $127 \pm 18$ & .88 \\
DBP $(\mathrm{mmHg})$ & $71 \pm 9$ & $75 \pm 13$ & .52 \\
Energy intake $(\mathrm{kcal} /$ day) & $1646 \pm 209$ & $1800 \pm 249$ & .22 \\
Protein intake $(\mathrm{g} /$ day) & $76 \pm 12$ & $77 \pm 13$ & .92 \\
Calcium intake $(\mathrm{mg} /$ day) & $661 \pm 148$ & $764 \pm 194$ & .27 \\
Vitamin D intake ( $\mu \mathrm{g} /$ day) & $22 \pm 7$ & $20 \pm 8$ & .68 \\
Vitamin K intake $(\mu \mathrm{g} /$ day) & $406 \pm 180$ & $448 \pm 237$ & .71 \\
\hline
\end{tabular}

Data are presented as mean \pm SD

$B M I$ body mass index, $S M I$ skeletal muscle mass index

$S B P$ systolic blood pressure, DBP diastolic blood pressure group interaction are shown. An interaction was found for pelvis BMD, and at follow-up, smaller and larger values were found in the control and training groups, respectively $(0.9 \%$ decrease and $1.6 \%$ increase, Fig. 2$)$, with marginal significance ( $p=0.189$ and 0.059 , respectively). Accordingly, the within-group change in pelvis BMD was significantly greater in the training group as compared to the control group $(p=0.042)$. No significant differences were found in within-group changes in total body BMD or BMD for other segments.

\section{Muscle strength and SMI}

A significant group $\times$ time interaction was found for KE strength (Table 3), and a significantly larger value at followup $(p=0.030)$ was found in the training group $(15.5 \%$ increase, Fig. 3). Accordingly, the within-group change in the training group was also significantly greater than that in the control group $(p=0.030)$. No significant main or interaction effects were found in grip strength or SMI.

\section{Discussion}

We assessed the effects of 6 weeks of low-repetition, lightload power training on BMD, SMI, and muscle strength in postmenopausal women with sarcopenia, and found that within-group changes in pelvis BMD and KE strength were significantly greater in the training group than in the control group. This suggests that the training significantly increased pelvis BMD and KE strength.

In contrast to previous power training studies reporting that regional BMD was maintained, not increased, at follow-up in early postmenopausal women $[8,9]$, we found a significant within-group increase in pelvis BMD in the training group relative to the control group. The previous studies used high-load power training, while we used light-load power training, which appeared to affect peak power output during training. Peak power during light-load power training was reportedly comparable or even larger than that of high-load power training [11]. Such a difference in peak power output may have led to the variation observed in BMD outcomes. Dynamic loading is required to stimulate appositional bone growth [18], and bone adaptation is known to be influenced by peak loading magnitude [18] and velocity of load $[19,20]$, suggesting that the most important factor for bone adaptation is peak power. Light-load power training may have helped our subjects generate greater power output than that generated by high-load power training, thereby facilitating an increase in BMD.

Performing low-repetition exercises with 15-s rest intervals may have also contributed to increases in BMD. Low-repetition protocols provide more rest intervals than high-repetition protocols, restoring the ability to generate muscle power [14] and allowing subjects to maximize muscle power throughout the training sessions. 
Table 2 Changes (mean \pm SD) in bone mineral density (BMD)

\begin{tabular}{|c|c|c|c|c|c|c|}
\hline & \multicolumn{2}{|l|}{ Training $(n=7)$} & \multicolumn{2}{|l|}{ Control $(n=8)$} & \multicolumn{2}{|c|}{ ANOVA Interaction } \\
\hline & Pre & Post & Pre & Post & $\bar{F}$ & $P$ \\
\hline \multicolumn{7}{|c|}{$\mathrm{BMD}\left(\mathrm{g} / \mathrm{cm}^{2}\right)$} \\
\hline Total body & $0.981 \pm 0.074$ & $0.981 \pm 0.063$ & $0.996 \pm 0.057$ & $0.992 \pm 0.061$ & 0.860 & 0.371 \\
\hline Arm & $0.681 \pm 0.042$ & $0.676 \pm 0.047$ & $0.693 \pm 0.042$ & $0.688 \pm 0.047$ & 0.035 & 0.854 \\
\hline Spine & $0.890 \pm 0.069$ & $0.883 \pm 0.051$ & $0.877 \pm 0.077$ & $0.870 \pm 0.079$ & 0.003 & 0.957 \\
\hline Pelvis & $0.920 \pm 0.076$ & $0.935 \pm 0.080$ & $0.926 \pm 0.072$ & $0.917 \pm 0.057$ & 6.061 & $0.029^{*}$ \\
\hline Leg & $0.985 \pm 0.090$ & $0.987 \pm 0.082$ & $1.026 \pm 0.069$ & $1.025 \pm 0.077$ & 0.213 & 0.652 \\
\hline
\end{tabular}

In fact, a previous study demonstrated that power training with a longer inter-set rest period was more effective for increasing power [21]. It has also been reported that a rest interval of at least $14 \mathrm{~s}$ is needed to restore the mechanosensitivity of bone cells and to enhance the osteogenic effects of exercise [16]. Considered collectively, low-repetition, light-load power training would allow individuals to have sufficient rest intervals required for maximizing power output and restoring mechanosensitivity of bone cells, thereby positively affecting BMD.

With the exception of pelvis BMD, no significant improvements were found in skeletal sites in the training group. Such region-specific increases in BMD may be due to the effects of aging as well as weight-bearing. Looker et al. [22] suggested that age-related loss in BMD and weight-bearing were both greatest in the pelvis compared to other sites. In fact, although not significant, reduced BMD in the pelvis was noted in the control group at follow-up as compared to baseline $(0.9 \%$ decrease, $p=0.189)$. Lower-limb power training in our study required concentric and eccentric muscle contractions of lower-limb muscles. Mechanical loading resulting from such muscle activities and weight-bearing forces may have facilitated the increase in pelvis BMD.

Knee extensor strength significantly improved by $15.5 \%$ in the training group, while no significant difference was observed in SMI. This result is consistent with results from another study that found a 16\% increase in leg extension strength after 10 weeks of light-load power training $(20 \%$ $1 R M, 8$ reps $\times 3$ sets) in elderly individuals [23]. Since a significant increase in muscle strength was observed without any increase in muscle mass, the increase is likely attributable to neural adaptations. Increases in muscle strength can be achieved without muscle hypertrophy because of neural adaptations in the early phase of training [24, 25]. Other studies have found that neural activation was greater with power training than with strength training $[25,26]$. Thus, low-repetition, light-load power training could be used to improve muscle function as well as BMD in postmenopausal women with sarcopenia.

The major limitations of this study were the small sample size and short training duration of the exercise intervention. A lack of significant differences in BMD for areas other than the pelvis, as well as SMI and grip

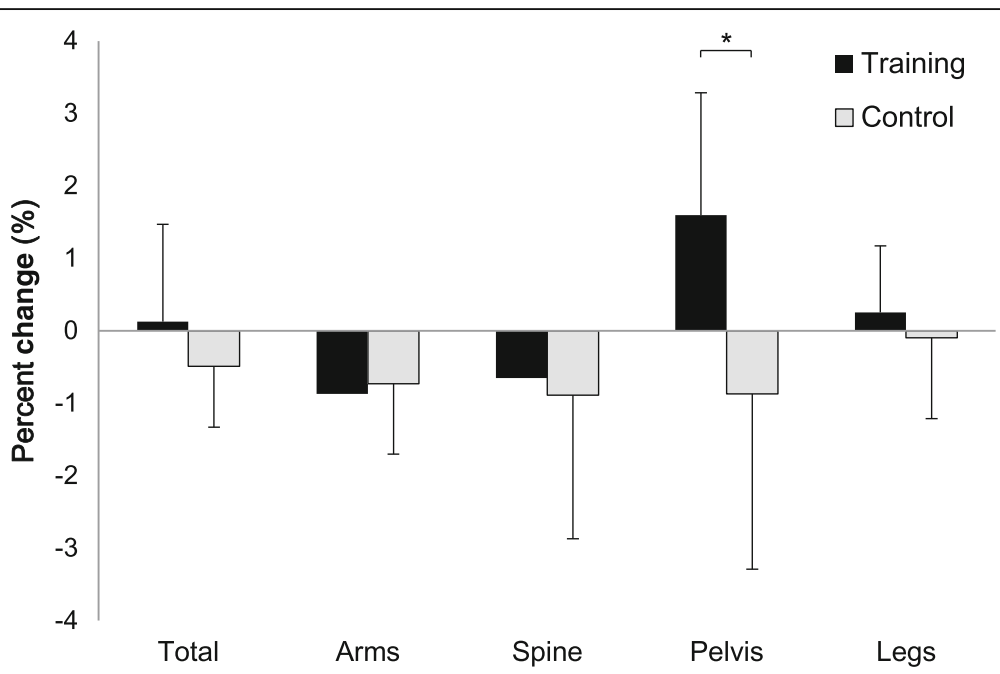

Fig. 2 Percent change in bone mineral density after 6 weeks of training. ${ }^{*}$ Significant difference between groups, $p<0.05$ 
Table 3 Changes (mean \pm SD) in strength parameters

\begin{tabular}{|c|c|c|c|c|c|c|}
\hline & \multicolumn{2}{|c|}{ Training $(n=7)$} & \multicolumn{2}{|c|}{ Control $(n=8)$} & \multicolumn{2}{|c|}{ ANOVA Interaction } \\
\hline & Pre & Post & Pre & Post & $\mathrm{F}$ & $P$ \\
\hline Grip strength (kg) & $24.3 \pm 3.1$ & $24.4 \pm 3.1$ & $25.7 \pm 3.9$ & $24.4 \pm 4.3$ & 2.748 & 0.121 \\
\hline KE strength $\left(\mathrm{N} / \mathrm{kg}^{2}\right)$ & $1.8 \pm 0.3$ & $2.0 \pm 0.3$ & $2.0 \pm 0.3$ & $2.0 \pm 0.4$ & 5.956 & $0.030^{*}$ \\
\hline SMI $\left(\mathrm{kg} / \mathrm{m}^{2}\right)$ & $5.4 \pm 0.3$ & $5.5 \pm 0.3$ & $5.6 \pm 0.5$ & $5.6 \pm 0.4$ & 1.044 & 0.326 \\
\hline
\end{tabular}

KE Knee-Extensor, SMI Skeletal muscle mass index

${ }^{*} p<0.05$

strength, may have been due to the small sample size. Furthermore, an intervention duration of 6 weeks may not be long enough to detect significant differences in $\mathrm{BMD}$, considering that other studies have indicated that significant improvements in BMD require at least 3 months to become apparent [27]. The small increase in pelvis BMD (1.6\%) observed after the exercise intervention could have been due to this shorter intervention period. That said, one previous study reported that inter-day reproducibility of pelvis BMD obtained from DXA was below 1.3\% [28], indicating that the $1.6 \%$ increase in pelvis BMD observed in our study would represent an actual change, while the $0.9 \%$ decrease in pelvis BMD observed in the control group appeared to be within expected measurement error. Although the decrease in the control group contributed to the significant time $\times$ group interaction in pelvis BMD, the present study demonstrated that even with 6 weeks of training, a significant relative increase could be observed at least in pelvis BMD and knee extensor strength. Future studies

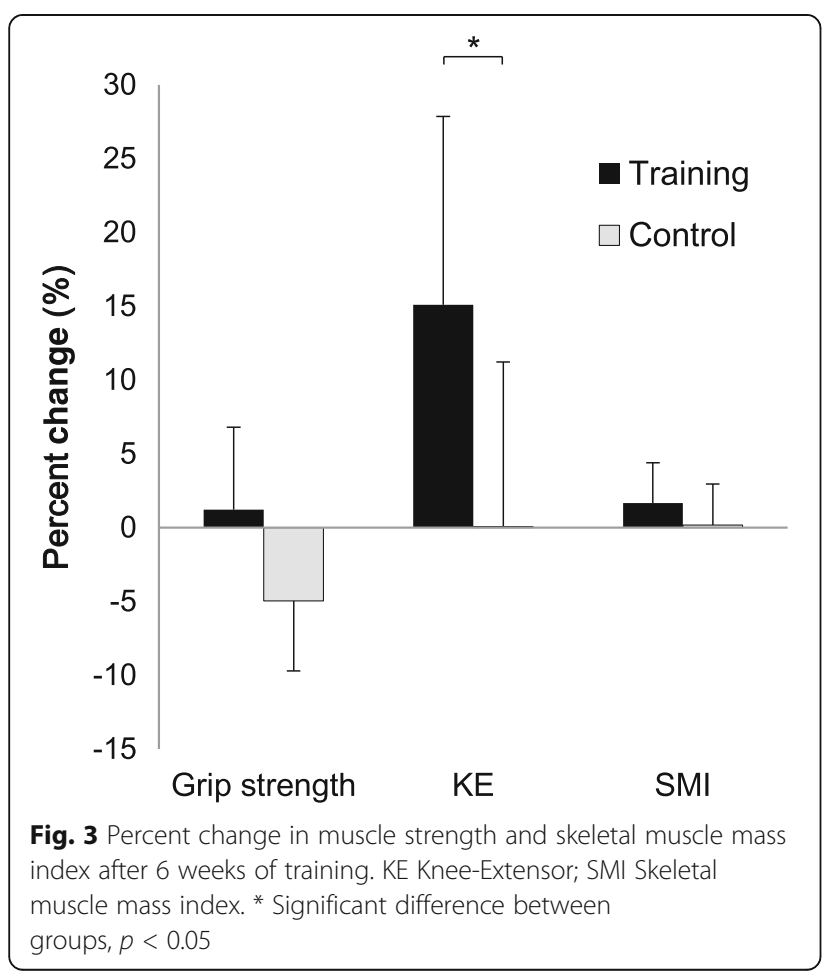

should investigate the long-term effects of our proposed power training program with a larger sample size.

\section{Conclusion}

This study provides the first evidence that low-repetition, light-load power training significantly increases pelvis BMD in postmenopausal women with sarcopenia. Since this training program does not require high-load exercise, high levels of adherence would be anticipated due to its ease of implementation. We conclude that low repetition, lightload power training would be an effective form of training exercise for sedentary adults who are at risk for osteoporosis and wary of heavy loads and/or fatigable training.

\section{Abbreviations}

BMC: Bone mineral content; BMD: Bone mineral density; DXA: Dual x-ray absorptiometry; FFM: Fat-free mass; KE: Knee extensor; Nm: Newton•meters; RM: Repetition maximum; SD: Standard deviation; SMI: Skeletal muscle mass index

\section{Acknowledgements}

We thank the subjects who participated in this study.

\section{Funding}

This work was supported by Grants-in-Aid for Scientific Research from the Ministry of Education, Culture, Sports, Science and Technology of Japan (KAKENHI: \#24300239 for K. Sanada). The funders played no role in study design, data collection, and analysis, decision to publish, or preparation of the manuscript.

Availability of data and materials

The datasets generated and analyzed during the current study are available from the corresponding author on reasonable request.

\section{Authors' contributions}

$\mathrm{KH}$ : conception and design of the study, supervision of the exercise program, acquisition of data, statistical analysis, and manuscript drafting and revising. TK: acquisition of data and manuscript drafting and revising. MF: acquisition of data and manuscript drafting and revising. Ml: acquisition of data. KSat: acquisition of data. TH: conception and design of the study and primary outcomes assessment. KSan: design and coordination of the study and manuscript drafting and revising. All authors read and approved the final manuscript.

Competing interests

The authors declare that they have no competing interests.

Consent for publication

Not applicable.

Ethics approval and consent to participate

This study was approved by the Ethics Committee of Ritsumeikan University (Approval number: BKC-IRB-2012-032).

\section{Publisher's Note}

Springer Nature remains neutral with regard to jurisdictional claims in published maps and institutional affiliations. 


\section{Author details}

College of Sport and Health Science, Ritsumeikan University, Shiga, Japan. ${ }^{2}$ Graduate School of Human Development and Environment, Kobe University, Kobe, Japan. ${ }^{3}$ Department of Sports Medicine for Health Promotion, Tokyo Medical University, Tokyo, Japan.

Received: 10 August 2016 Accepted: 21 April 2017

Published online: 02 May 2017

\section{References}

1. Sambrook PN, Cameron ID, Chen JS, Cumming RG, Lord SR, March LM, Schwarz J, Seibel MJ, Simpson JM. Influence of fall related factors and bone strength on fracture risk in the frail elderly. Osteoporos Int. 2007;18(5):603-10.

2. Bliuc D, Nguyen TV, Eisman JA, Center JR. The impact of nonhip nonvertebral fractures in elderly women and men. J Clin Endocrinol Metabolism. 2014;99(2):415-23.

3. Asikainen TM, Kukkonen-Harjula K, Miilunpalo S. Exercise for health for early postmenopausal women - a systematic review of randomised controlled trials. Sports Med. 2004;34(11):753-78.

4. ROSENBERG. Epidemiologic and methodologic problems in determining nutritional status of older persons. Proceedings of a conference. Albuquerque, New Mexico, October 19-21. Am J Clin Nutr 1989. 1988:50(5 Suppl):1121-235.

5. Cruz-Jentoft AJ, Baeyens JP, Bauer JM, Boirie Y, Cederholm T, Landi F, Martin FC, Michel JP, Rolland Y, Schneider SM, et al. Sarcopenia: European consensus on definition and diagnosis: report of the European working group on sarcopenia in older people. Age Ageing. 2010;39(4):412-23.

6. Landi F, Liperoti R, Russo A, Giovannini S, Tosato M, Capoluongo E, Bernabei $R$, Onder $G$. Sarcopenia as a risk factor for falls in elderly individuals: results from the ilSIRENTE study. Clin Nutr. 2012;31(5):652-8.

7. Verschueren S, Gielen E, O'Neill TW, Pye SR, Adams JE, Ward KA, Wu FC, Szulc P, Laurent M, Claessens F, et al. Sarcopenia and its relationship with bone mineral density in middle-aged and elderly European men. Osteoporosis Int. 2013;24(1):87-98.

8. Stengel SV, Kemmler W, Pintag R, Beeskow C, Weineck J, Lauber D, Kalender WA, Engelke K. Power training is more effective than strength training for maintaining bone mineral density in postmenopausal women. J Appl Physiol. 2005;99(1):181-8.

9. Mosti MP, Kaehler N, Stunes AK, Hoff J, Syversen U. Maximal strength training in postmenopausal women with osteoporosis or osteopenia. J Strength Cond Res. 2013;27(10):2879-86.

10. Cuoco A, Callahan DM, Sayers S, Frontera WR, Bean J, Fielding RA. Impact of muscle power and force on gait speed in disabled older men and women. J Gerontol A Biol Sci Med Sci. 2004;59(11):1200-6.

11. Mohamad NI, Cronin JB, Nosaka KK. Difference in kinematics and kinetics between high- and low-velocity resistance loading equated by volume: implications for hypertrophy training. J Strength Cond Res. 2012;26(1):269-75.

12. Sleivert $\mathrm{G}$, Taingahue M. The relationship between maximal jump-squat power and sprint acceleration in athletes. Eur J Appl Physiol. 2004;91(1):46-52.

13. Snow CM, Shaw JM, Winters KM, Witzke KA. Long-term exercise using weighted vests prevents hip bone loss in postmenopausal women. J Gerontol a-Biol. 2000; 55(9):M489-91.

14. Paulo CA, Roschel H, Ugrinowitsch C, Kobal R, Tricoli V. Influence of different resistance exercise loading schemes on mechanical power output in work to rest ratio - equated and - nonequated conditions. J Strength Cond Res. 2012;26(5):1308-12.

15. Sanada K, Miyachi M, Tanimoto M, Yamamoto K, Murakami H, Okumura S, Gando Y, Suzuki K, Tabata I, Higuchi M. A cross-sectional study of sarcopenia in Japanese men and women: reference values and association with cardiovascular risk factors. Eur J Appl Physiol. 2010;110(1):57-65.

16. Robling AG, Burr DB, Turner $\mathrm{CH}$. Recovery periods restore mechanosensitivity to dynamically loaded bone. J Exp Biol. 2001;204(Pt 19):3389-99.

17. Jayaraman A, Thompson CK, Rymer WZ, Hornby TG. Short-term maximalintensity resistance training increases volitional function and strength in chronic incomplete spinal cord injury: a pilot study. J Neurol Phys Ther. 2013;37(3):112-7.

18. Robling AG, Duijvelaar KM, Geevers JV, Ohashi N, Turner CH. Modulation of appositional and longitudinal bone growth in the rat ulna by applied static and dynamic force. Bone. 2001;29(2):105-13.

19. Turner $\mathrm{CH}$. Three rules for bone adaptation to mechanical stimuli. Bone. 1998;23(5):399-407.
20. Oconnor JA, Lanyon LE, Macfie $H$. The Influence of strain rate on adaptive bone remodeling. J Biomech. 1982;15(10):767-81.

21. Pincivero DM, Campy RM. The effects of rest interval length and training on quadriceps femoris muscle. Part I: knee extensor torque and muscle fatigue. J Sport Med Phys Fit. 2004;44(2):111-8.

22. Looker AC, Melton LJ 3rd, Harris T, Borrud L, Shepherd J, McGowan J. Age, gender, and race/ethnic differences in total body and subregional bone density. Osteoporos Int. 2009;20(7):1141-9.

23. de Vos NJ, Singh NA, Ross DA, Stavrinos TM, Orr R, Singh MAF. Optimal load for increasing muscle power during explosive resistance training in older adults. J Gerontol a-Biol. 2005;60(5):638-47.

24. Hakkinen K, Kraemer WJ, Newton RU, Alen M. Changes in electromyographic activity, muscle fibre and force production characteristics during heavy resistance/power strength training in middle-aged and older men and women. Acta Physiol Scand. 2001;171(1):51-62.

25. Sale DG. Neural adaptation to resistance training. Med Sci Sports Exerc. 1988;20(5 Suppl):S135-45.

26. Sale DG. Influence of exercise and training on motor unit activation. Exerc Sport Sci Rev. 1987;15:95-151.

27. Simpson AH, Murray IR. Osteoporotic fracture models. Current Osteoporos Rep. 2015;13(1):9-15

28. Franck $H$, Munz $M$. Total body and regional bone mineral densitometry (BMD) and soft tissue measurements: correlations of BMD parameter to lumbar spine and hip. Calcif Tissue Int. 2000:67(2):111-5.

\section{Submit your next manuscript to BioMed Central and we will help you at every step:}

- We accept pre-submission inquiries

- Our selector tool helps you to find the most relevant journal

- We provide round the clock customer support

- Convenient online submission

- Thorough peer review

- Inclusion in PubMed and all major indexing services

- Maximum visibility for your research

Submit your manuscript at www.biomedcentral.com/submit
Biomed Central 